\title{
TO THE 110th ANNIVERSARY OF ACADEMICIAN M.M. BOGOLYUBOV BIRTHDAY
}

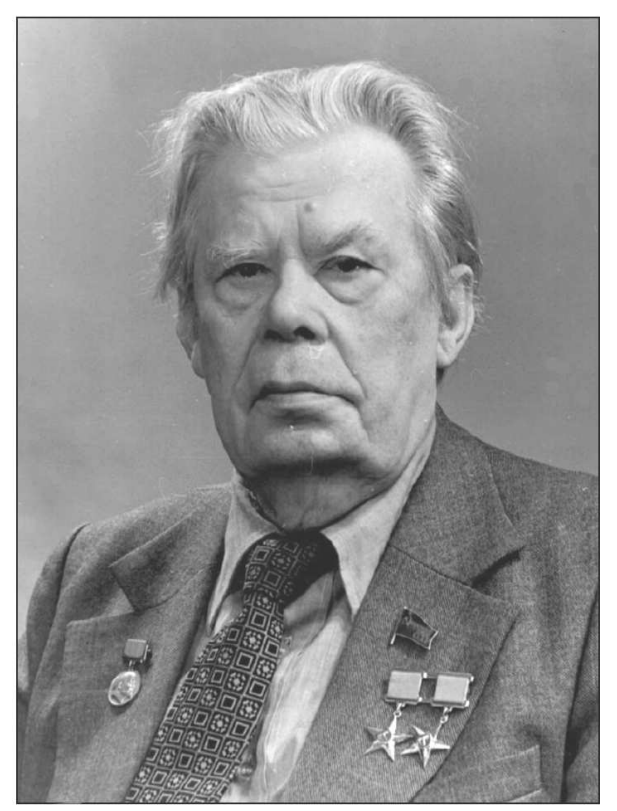

The 110th anniversary of Mykola Bogolyubov provides a good opportunity to recollect the scientific achievements of this brilliant scientist and to discuss once again his invaluable contribution to the development of various fields of theoretical and mathematical physics - nonlinear mechanics, nuclear physics, quantum field theory, high-energy physics, condensed matter physics, etc. The departments, research groups, and even whole institutes founded by him continue to work nowadays. The Bogolyubov scientific school, which has grown on his ideas, is successfully developing already in its fourth generation.

Mykola Bogolyubov was born on August 21, 1909 in Nizhny Novgorod. That very year the Bogolyubov family moved from Nizhny Novgorod to Nizhyn, Chernihiv province, where Mykola's father - by that time already known theologist Mykola Mykhailovych
Bogolyubov - got the position of Professor of scripture at the Prince Bezborodko Historical and Philological Institute. It should be noted that this institute had a long educational traditions and a high reputation. Mykola Hohol, Yevhen Hrebinka, Leonid Hlibov, and many other outstanding figures had been its students. Four years later, Mykola Mykhailovych became a professor of theology at St. Vladimir' University and the family moved to Kyiv.

In 1917, when Bogolyubov was eight, he entered the preparatory class of the First Alexander Classic Kyiv Gymnasium, but he studied there for less than two years. In 1920, Soviet power was finally established in Kyiv, the Department of Theology was closed, and Mykola's father was forced to take a parish in the village of Velyka Krucha, Poltava province. Mykola began to attend the Velykokruchans'ka seven-year school and graduated in 1922. When recollecting this school, Bogolyubov said that it's pedagogical team would be honor to the best schools of the capital. By the way, the certificate on the graduation of the sevenyear school was the only document on education that Bogolyubov received in his life, and the words about his officially received education are "I became a scientist in Velika Krycha". Due to school and home education, at the age of 13 Mykola Bogolyubov had knowledge at the graduate level of the faculty of physics and mathematics of the university.

In 1922, the Bogolyubov family returned to Kyiv. Bogolyubov's father asked the famous mathematician Academician Dmytro Grave for advise concerning the further education of his elder son. Professor Grave, after acquaintance with young Bogolyubov, told his father that attending lectures at any university would make no sense for the young man, but advised to continue education individually. Since then Bogolyubov began to participate in the seminars of

ISSN 2071-0194. Ukr. J. Phys. 2019. Vol. 64, No. 8 
Academician Grave. In the spring of 1923 young Bogolyubov met his teacher, mentor, and future colleague Academician Mykola Krylov, who began to give special classes in mathematics and mechanics for Bogolyubov. When Mykola Bogolyubov was 15 years old, he published his first scientific paper, and on June 1, 1925, a special decree was adopted by the Ukrhlavnauka, which stated: "In view of phenomenal gift for mathematics, to consider M. Bogolyubov as a postgraduate student of the Department of Mathematics since July 18, 1925. Add him to the payroll list".

In 1930, Bogolyubov received his first recognition he got the Award of the Academy of Sciences of Bologna (Italy). The same year, namely, on April 6, 1930, at the General Meeting of the Department of Physics and Mathematics of the All-Ukrainian Academy of Sciences (VUAN), on the recommendation of D. Grave and M. Krylov, the degree of Doctor of Sciences was awarded to Bogolyubov without thesis. Academic title of Professor on the Department of Theory of Functions was conferred on Bogolyubov in 1936 after he had began teaching at Kyiv University. Later, in 1939, M. Bogolyubov was elected a corresponding member, in 1948 - Academician of the Academy of Sciences of the Ukrainian SSR. In 1947, he became a Corresponding Member of the Academy of Sciences of the USSR, and in 1953 he became its full member.

The topmost results of Bogolyubov in 1932-37 include the foundation, together with his teacher, of a new section of mathematical physics - the theory of nonlinear oscillations, that later would be called nonlinear mechanics. In particular, they have developed new methods for integrating nonlinear differential equations describing vibration processes. These results have been summarized in many joint monographs by Bogolyubov and Krylov of this period. Among them are "On Some Formal Decompositions at Nonlinear Mechanics", "New Methods of Nonlinear Mechanics", "Application of Methods of Nonlinear Mechanics to the Theory of Stationary Oscillations", "Introduction to Nonlinear Mechanics". In 1955, a fundamental monograph by Bogolyubov and Mitropolsky "Asymptotic Methods in the Theory of Nonlinear Oscillations" was published.

In 1935-1936, Bogolyubov represents the Department of Mathematical Physics of VUAN abroad. He gives lectures on the theory of nonlinear oscillations at Henri Poincaré Institute in France, Belgian Math-

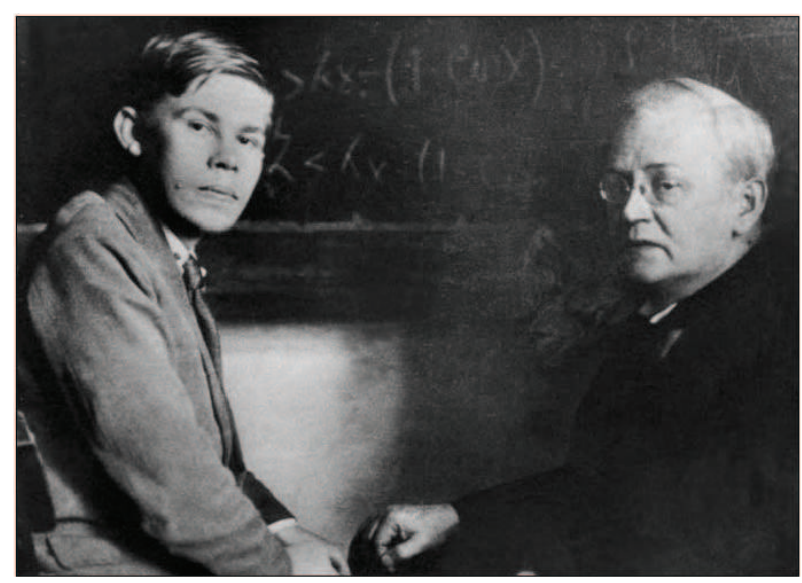

Fig. 1. M.M. Bogolyubov and Academician M.M. Krylov

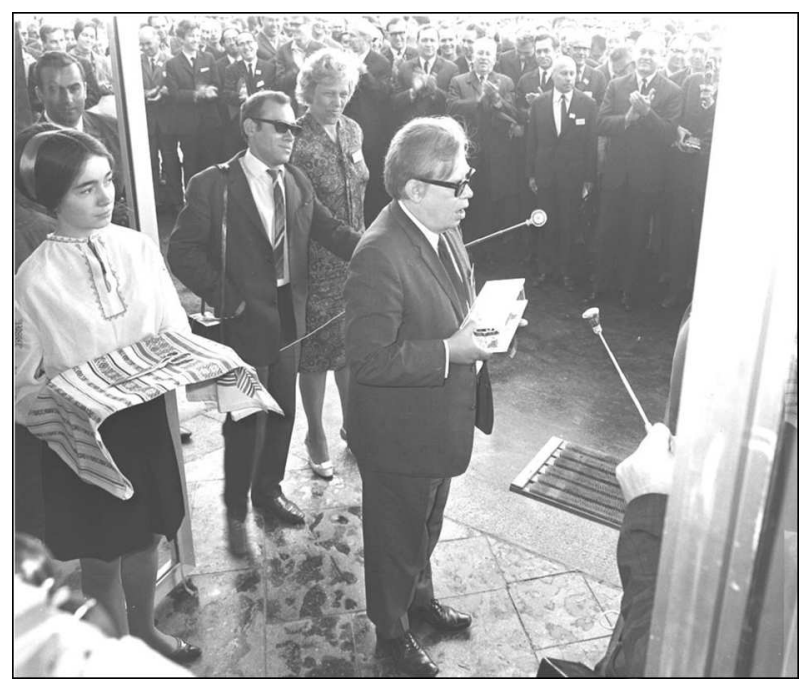

Fig. 2. Opening of a new building of the Institute for Theoretical Physics

ematical Society, and Belgian Research Institute. In 1940, after the unification of the Northern Bukovyna with Ukraine Bogolyubov participated in the formation of the mathematical department at the Physics and Mathematics Faculty of Chernivtsi University.

In summer of 1941, M. Bogolyubov was evacuated to Ufa and then sent to Moscow. At this time, as Mykola Mykolayovych writes in his autobiography, he, while continuing theoretical studies in nonlinear mechanics, was mainly concerned with defense topics. Bogolyubov returned to Kyiv in early 1944.

One of the most fruitful periods of creativity of Bogolyubov is associated with Kyiv. Just here 


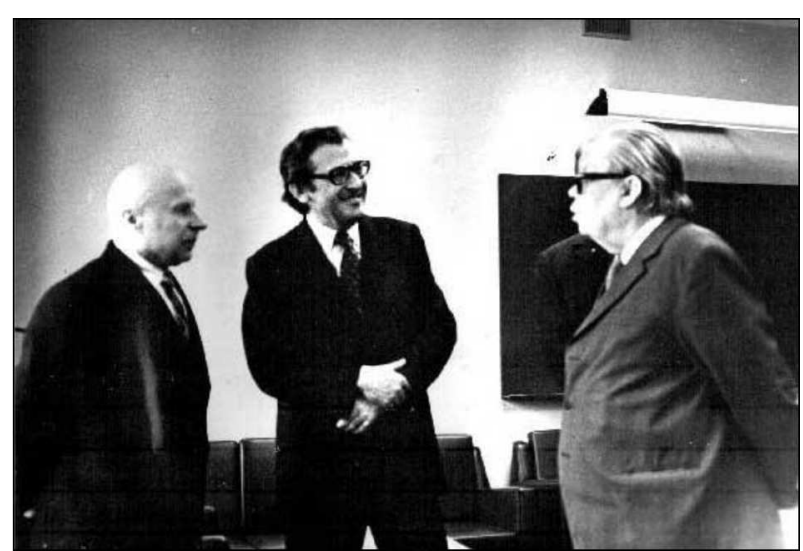

Fig. 3. Academicians O.S. Davydov, O.G. Sitenko, and M.M. Bogolyubov after the opening of a new building of the Institute (1970)

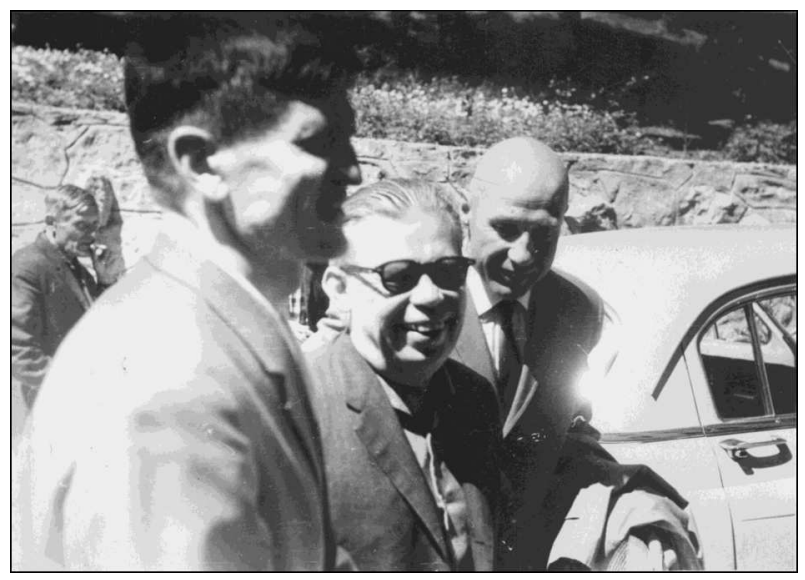

Fig. 4. M.M. Bogolyubov and his disciples, Academicians O.S. Parasyuk (left) and Yu.O. Mytropol's'kyi (right)

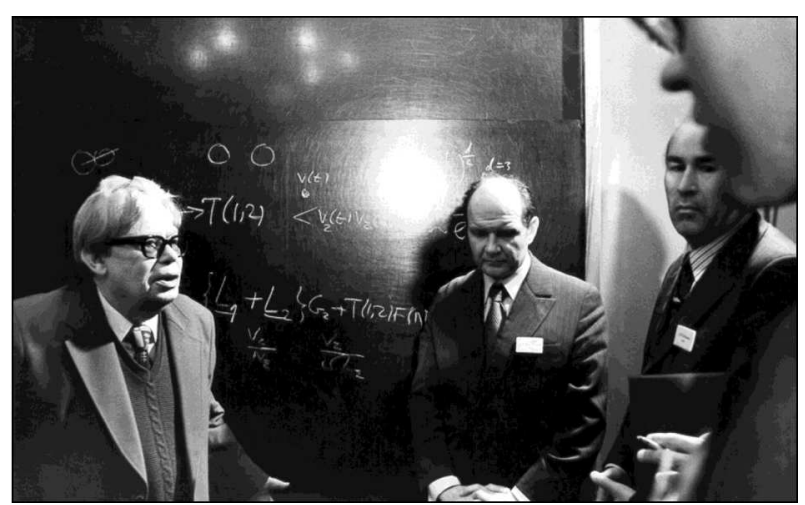

Fig. 5. M.M. Bogolyubov with Academicians I.R. Yukhnovskyi and D.Ya. Petryna
M. Bogolyubov initiated new fields of theoretical and mathematical physics, in particular, wrote his classical works on modern statistical theory. Particularly, in 1946, M. Bogolyubov published the worldfamous book "Problems of the Dynamic Theory in Statistical Physics". The results given in this work have initiated a new stage in the progress of statistical mechanics after the achievements related to Maxwell, Boltzmann, Gibbs. Bogolyubov proposed a dynamic approach to the formulation of the kinetic theory based on the chain of equations for equilibrium and nonequilibrium many-particle distribution functions - the chain of Bogolyubov-Borne-GreenKirkwood-Yvon equations (it should be noted that Bogolyubov gave the most general and mathematically rigorous chain derivation). Using a small-scale expansion of this chain and applying the assumption of the existence of a hierarchy of time scales (known in the world literature as the hierarchy of Bogolyubov's characteristic times), Bogolyubov obtained closed kinetic equations for one-particle distribution functions not only for neutral gas but also for plasma. The latter equation today is called the Bogolyubov-BalescuLennard kinetic equation. Instead of Boltzmann's hypothesis of molecular chaos, he proposed the principle of complete weakening of initial correlations (Bogolyubov's principle), which made it possible to calculate collision integrals on the basis of a reduced chain of equations for distribution functions. To describe the next stage in the evolution of the system, Bogolyubov obtained the equations of hydrodynamics.

1947 - another brilliant result: the microscopic theory of superfluidity. The article in which this theory was formulated has remained, for many years, one of the most cited works of our time. In this work, Bogolyubov for the first time applied a new mathematical technique known today as Bogolyubov's canonical transformation. On the example of a weakly idealized Bose gas, Bogolyubov explained from the first principles the formation of the excitation spectrum of a superfluid helium and thus the nature of this macroscopic quantum phenomenon. He later summarized his mathematical formalism for the foundation of a microscopic theory of superconductivity. Bogolyubov perfectly studied the methods of secondary quantization for quantum statistical systems. His "Lectures on Quantum Statistics", published in 1949, could be a good illustration of effective application of this method to quantum statistics. This contributed to 
his interest in the problems of quantum field theory, where he also managed to obtain a number of outstanding results. A brilliant example is the development of a method for eliminating divergences in the quantum field theory based on the use of the subtraction procedure, and proving one of the central theorems of the renormalization theory, known as the Bogolyubov-Parasyuk theorem. The discovery of the general form of the subtraction procedure and its justification were of great importance for the further development of high-energy physics. It made it possible, in particular, to prove the renormality of a unified theory of electroweak interactions, as well as of supersymmetric theories, to obtain operator expansions at short distances, to study phase transitions, and so on.

In 1951-1953, Mykola Bogolyubov worked at the top-secret object of the Soviet Union - "Arzamas-16" (Sarov), as well as at the Institute of Atomic Energy (now "Kurchatov Institute" in Moscow), where, in parallel with the mathematical studies of the problems related to the creation of hydrogen weapons, he worked on the problems concerning the magnetic fusion reactor. It should be noted that the results obtained then by Bogolyubov in the field of nuclear fusion have not been published, since they were a part of secret reports. Only after removing the mark of secrecy from these results it turned out to be that a considerable part of the results on the kinetic plasma theory had been obtained by Bogolyubov before they were obtained and published independently by other authors in open literature.

From 1948, M. Bogolyubov along with his work in Kyiv began to head the Department of Mathematical Physics at the Institute of Chemical Physics in Moscow, and from 1949 - also the Department of Theoretical Physics of the Steklov Mathematical Institute of the Academy of Sciences of the USSR. In 1956, Mykola Bogolyubov became the Director of the Laboratory of Theoretical Physics of the Joint Institute for Nuclear Research (JINR) in Dubna. In January 1965, at the session of the plenipotentiaries of the governments of the member states of the Institute, Mykola Bogolyubov was elected the Director of JINR, which he has headed for over 20 years. Since 1957, Mykola Bogolyubov also headed the Laboratory of the Theory of Atomic Nuclei and Elementary Particles at the Institute of Physics of the Academy of Sciences of the Ukrainian SSR.

ISSN 2071-0194. Ukr. J. Phys. 2019. Vol. 64, No. 8
Among other results by Bogolyubov concerning the perturbation methods in the quantum field theory, one should also mention the method of renormalization group - the new general approach in theoretical physics, which has found application in various fields.

Mykola Bogolyubov is a founder of a new field of research in the quantum theory that was later called the axiomatic field theory. In particular, the proposed derivation of the dispersion relations has led to the development of a new mathematical approach to the analytic continuation of the generalized functions of many variables. For these studies in 1966, Mykola Bogolyubov was awarded the Danny Heinemann Award. In his welcome address, Professor R. Jost said: "You made an unforgettable impression on me. Most theorists at the time were disrespectful of mathematics, and logical deduction was "trampled". Only the romantic influence of genius could have value. And then you appeared, a person who knows both mathematics and physics and who is ready to solve complex problems that require their logical combination. It seems to me that this is a reflection of the national character of your great people".

In 1961, M. Bogolyubov introduced the fundamental concept of quasiaverages and thus, in fact, a new theory of phase transformations was created. The spread of these ideas to the physics of elementary particles was called spontaneous symmetry breaking another fundamental result of Mykola Bogolyubov, which is important for quantum physics.

During the period of 1964-1966, Bogolyubov published important papers on the symmetry theory and quark models of elementary particles. One of the important results in this field is the introduction of the new quantum number for quarks, now known as color, proposed by him and his disciples A.N. Tavkhelidze and B.V. Struminskii for quarks. Now this parameter is known as color.

Bogolyubov's scientific activity revealed the unity of the mathematical structure of theories for different branches of physics. The follower of Bogolyubov Academician V. Vladimirov noted: "Combination of mathematics and physics in the works of M.M. Bogolyubov made it possible for him to contribute considerably to the development of theoretical physics and in fact to create the foundations of modern mathematical physics, which continues the traditions of Hilbert, Poincare, Einstein, Dirac". 


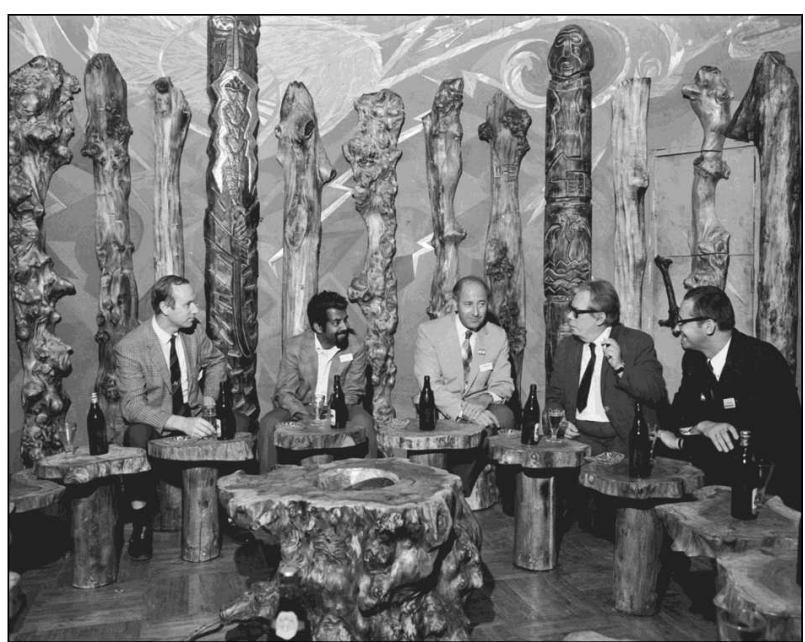

Fig. 6. E.C.G. Sudarshan (USA), R.E. Marshak (USA), M.M. Bogolyubov, and V.P. Shelest at the Institute for Theoretical Physics during the XV International Conference on High Energy Physics (Kyiv, 1970)

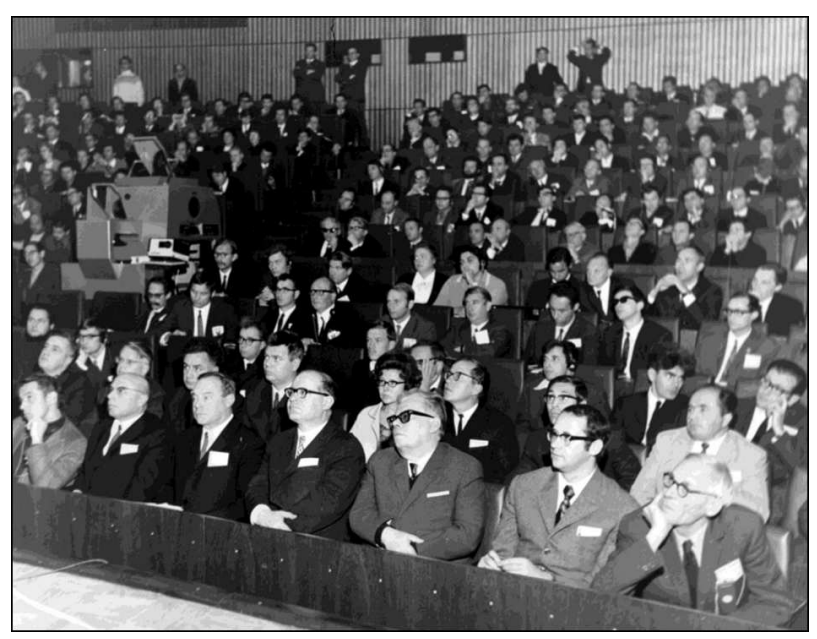

Fig. 7. First International Conference on Plasma Theory (Kyiv, Institute for Theoretical Physics of the Academy of Sciences of the UkrSSR, 1971)

Mykola Bogolyubov had the talent of a great researcher and outstanding organizer of science. An example confirming his organizational skills is the foundation in 1966 of the Institute for Theoretical Physics that since 1993 is called by his name. It should be noted that the creation of an elite physical institute in Kyiv was an extremely difficult task. There were several reasons. These include the existence in the USSR of the Institute for Theoretical Physics of the USSR
Academy of Sciences in Chornogolovka (now the Landau Institite for Theoretical Physics of RAS), and the inconsistency to the "general line", according to which the priority in the development of fundamental research belonged obviously not to Ukraine, and problems with the formation of highly skilled staff capable to perform competitive research. It was necessary to have the influence and weight of Mykola Bogolyubov to succeed. It was also important that the First Secretary of the Communist Party of Ukraine Petro Shelest and President of the Academy of Sciences of Ukrainian SSR Borys Paton gave him great help and assistance in this matter. As a result of their joint efforts, on January 5, 1966, the Council of Ministers of the Ukrainian SSR adopted a decree "On the Establishment of the Institute for Theoretical Physics of the Academy of Sciences of the Ukrainian SSR", and in 1970, during the Rochester Conference, a new building of the Institute was opened on the site chosen by Bogolyubov.

Everything related to the foundation of the Institute has been done with the direct participation of Bogolyubov - from the choice of the site for the institute building to staff appointments. He formulated the main fields of scientific activity of the Institute, namely: elementary particle theory, theory of nuclei and nuclear reactions, and statistical physics. Mykola Bogolyubov invited outstanding scientists to the Institute, including his talented students. Among the scientists with world names whom he invited were Academicians O. Davydov, A. Petrov, O. Sitenko, I. Yukhnovsky; students of Bogolyubov: A. Tavhelidze (later Academician of the Russian Academy of Sciences), Academicians of the NAS of Ukraine O. Parasyuk, D. Petryna, Corresponding Member of the Academy of Sciences of Ukraine V. Shelest and others. As a result, for the first seven years of the directorship of Mykola Bogolyubov, the Institute has become a powerful center of theoretical physics, well-known not only in Ukraine, but also far beyond its borders.

Bogolyubov paied much attention to the development of international cooperation, in particular the organization of international conferences such as Rochester Conference on high-energy physics and international conferences on plasma theory initiated by him together with O. Sitenko. These conferences proved to be so successful that they were called the "Kyiv Conferences on Plasma Theory" and were held under this name in many countries around the world, 
periodically returning to Kyiv in 1976, 1987, and 2006.

As already mentioned, the scientific fields formulated by Mykola Bogolyubov have determined the activities of the Institute for many years. Today its main activities are related to high-energy physics and astrophysics, nucleus theory, quantum field theory, symmetry theory, the theory of nonlinear phenomena in condensed matter and plasmas, as well as the kinetic theory of highly nonequilibrium processes. In fact, this corresponds to somewhat extended trends formulated by Bogolyubov. We can say that much of the research activities of the Institute are related to the application and development of the ideas of Mykola Bogolyubov. In particular, in the field of theoretical high-energy physics, this concerns the dynamic generation of masses, spontaneous symmetry breaking, quantum chromodynamics, and the application of symmetry theory in quantum field theory. The same concerns Bogolyubov's ideas in the kinetic theory. As noted above, Mykola Bogolyubov is one of the founders of the theory of many-particle systems. Previously, such theory was used to describe gases and plasma.

But Bogolyubov's methods have also proved to be efficient for describing much more complex systems, in particular for the study of dusty plasmas, i.e., a mixture of plasmas and solid particles. Creative inheritance of Bogolyubov is also used today to solve the problems of condensed matter physics. These include the description of high-temperature superconductivity, the phenomenon of Bose condensation in various systems, nonlinear phenomena in solids and liquids, transport processes in molecular systems, and the kinetics of electron transport in nanoobjects. Methods of quantum field theory are, in turn, widely used in the study of low-dimensional and socalled Dirac structures, as well as new materials.

Along with scientific research and organizational activities, Bogolyubov carried out impressive pedagogical work. In 1936-1941 and 1944-1949, he taught at Kyiv State University, in 1945-1948 he was Dean of the Faculty of Mechanics and Mathematics, where he founded and headed the Department of Mathematical Physics. From November 1943, he was Professor at the Lomonosov Moscow University. In January 1953, Bogolyubov was elected the Head of the Department of Theoretical Physics of the University, where, in 1966, he also founded the well-known
Bogolyubov Department of Quantum Statistics and Field Theory.

It is important to note that Mykola Bogolyubov's life and work from the first years till the last days were closely connected with Ukraine. Being ethnic Russian by origin, he was brought up in the atmosphere of deep love to Ukraine, he felt great respect for the land where his childhood and adolescence passed, where he made his first steps in science and gained worldwide name. Desiring to share the fate of the Ukrainian people in everything, he considered himself Ukrainian, as he wrote about himself in all questionnaires and personal papers. The same entry was in his Soviet passport. Mykola Mykolayovych's attitude to Ukraine is comprehensively characterized by Alexey Bogolyubov's words about his elder brother: "Mykola Mykolayovych had two homelands - Russia and Ukraine and two native languages - Russian and Ukrainian. Beginning from the Velyka Crucha years, he became associated with Ukraine, and Shevchenko's poetry was, in fact, the first poetry he became interested in. The young graduate student of the Department of Mathematical Physics wrote the minutes of the seminars of the department in Ukrainian, and his first works were also written in Ukrainian." Further: "Mykola Mykolayovych, in the difficult times for Ukraine, when the Ukrainian intelligentsia started to be destroyed, when the shameful process of the Ukrainian Liberation Union took place in Kharkiv and Ukrainian books burned, he admitted himself to be a Ukrainian and so considered himself for his whole life. It is an indisputable fact that all the development of his personality and the acquisition of features of scientific creativity took place in Ukraine, and were also closely associated with Ukraine. He used to call Kyiv his favorite city, equating to him only Paris". Although these words are well known and have been cited for many times in articles about the Ukrainian period of Bogolyubov and memories of him, we have to mention them here, because they reveal the origins of Bogolyubov's love for Ukraine. Mykola Mykolayovych's attitude to his native Ukrainian land, to the Ukrainian language, should be a good example for many of our compatriots.

Mykola Bogolyubov passed away on February 13, 1992. He has left invaluable scientific heritage, numerous scientific schools, a large cohort of students and followers, with whom he always shared scientific ideas and interesting research. 


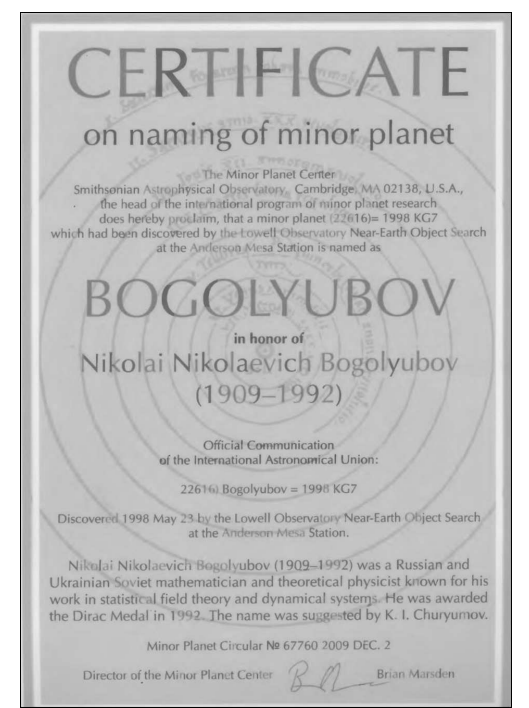

Fig. 8. Certificate on naming of a minor planet in the solar system as Bogolyubov

M.M. Bogolyubov was a scholar of wide international recognition. He was elected a member of 10 foreign academies of sciences and was awarded the honorary doctorate of 10 foreign universities. Foreign state and scientific awards also testify to the recognition of Bogolyubov's contribution to the world science. In particular, he is a winner of the Prize of the Academy of Sciences of Bologna (1930, Italy), Heinemann Prize of the American Physical Society (1966), Helmholtz Gold Metal (1969), Max Planck Prize of the Physical Society of Germany (1978), Franklin Prize (1974, USA), Prize of the Slovak Academy of Sciences (1975), Paul Dirac Prize (1992), and others.

In 1987, the International Center for Theoretical Physics in Trieste founded the Bogolyubov Prize for outstanding achievements in mathematics and solid state physics for scientists from developing countries. The National Academy of Sciences of Ukraine has also established the Bogolyubov Prize for the research in mathematics and physics. The Russian Academy of Sciences founded in 1999 the Bogolyubov
Gold Medal, for researches in the fields of mathematical physics and mathematics. The Bogolyubov Gold Medal was also founded in JINR. In 2018, the Bogolyubov Institute for Theoretical Physics started awarding Bogolyubov Prizes for the best works in theoretical and mathematical physics.

The monuments of Academician Bogolyubov were erected in Nizhny Novgorod and Dubna, and his busts were located in Kyiv at the Bogolyubov Institute for Theoretical Physics and at the JINR Laboratory of Theoretical Physics. A memorial plaque honoring Mykola Bogolyubov decorates the Red Building of Taras Shevchenko National University of Kyiv. A memorial sign in honor of Mykola Mykolayovych was erected in the village of Velyka Krucha.

The 100th anniversary of Bogolyubov birth is widely celebrated in Ukraine. The International Bogolyubov Conference "Modern Problems of Theoretical and Mathematical Physics" and the II Ukrainian Mathematical Congress were held in Kyiv, and the anniversary Bogolyubov Conference was also held in Lviv; books and articles about the life and work of the great scientist were published. The anniversary coin and the Bogolyubov Medal of the Ukrainian Mathematical Congress were minted.

On December 3, 2009, at the application of the famous Ukrainian astronomer K.I. Churyumov initiated by the Bogolyubov Institute for Theoretical Physics, the International Astronomical Union adopted the decision to give the minor planet of the Solar System $(22616)=1998$ KG7 the name Bogolyubov.

A brilliant scientist continues his life in the works of his students and numerous followers, including those who work at the Bogolyubov Institute for Theoretical Physics, and we are sure that the ideas of Mykola Bogolyubov will inspire many theorists for many years.

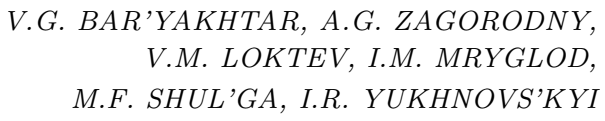

